\title{
La réadaptation en médecine interne
}

\section{Stephan Eberhard}

Président du groupe de travail de réadaptation en médecine interne de la SSMI

\section{* Groupe de travail de réadaptation en médecine} interne:

Dr Jürg Berchtold, Reha

Chrischona, Bürgerspital Basel Dr Werner Karrer,

Clinique Lucernoise Montana Dr Susi Stöhr, SUVA Luzern PD Dr Stefan Bachmann,

Kliniken Valens

Dr Patrick Konietzny,

Klinik Adelheid, Unterägeri

Dr Nic Zerkiebel,

Klinik Susenberg Zürich

Dr Dieter Breil,

Kantonsspital Olten

Dr Wanda Bosshard,

Hôpital de Lavaux

Prof. Dr Anne-Françoise Allaz, Hôpital Universitaire Genève Dr Bernard Davy,

Clinique La Lignière, Gland Dr Olivier Berclaz,

Clinique Bois-Bougy, Nyon Dr Josef Perseus, Zürcher

Höhenklinik Davos Clavadel

Correspondance:

Dr Stephan Eberhard MBA Clinique Bernoise Montana CH-3963 Crans-Montana

eberhard.stephan[at] bernerklinik.ch
La réadaptation est un élément important de la médecine moderne qui prend de plus en plus d'ampleur. Vu les comorbidités qui touchent un nombre de plus en plus élevé de patients en réadaptation, le spécialiste en médecine interne générale, ainsi que le secteur de la réadaptation en médecine interne, ne cessent de gagner en importance [1]. Le groupe de travail de réadaptation en médecine interne de la SSMI, dans la continuité de l'article du Dr Werner Karrer, concernant la réadaptation en médecine interne et publié en 2007 dans le Bulletin des médecins suisses [2], vous présente ce nouveau document. Il propose des recommandations et devrait servir de base de discussion pour les futurs développements du secteur de la réadaptation en médecine interne.

Grâce à sa formation, le spécialiste en médecine interne générale est capable de diagnostiquer la plupart des maladies de la personne adulte, de les traiter, de garantir les traitements à long terme, et cela de manière autonome ou à l'aide de spécialistes [3].

Il est non seulement la plaque tournante du processus diagnostic, mais aussi garant de la cohérence entre les différents avis spécialisés. La médecine de réadaptation suppose une approche qui a comme objectif de maintenir ou d'améliorer non seulement les capacités, mais aussi d'impliquer le patient dans les procédures de soin. C'est le couple «capacité-implication» qui facilite l'intégration du patient dans son milieu de vie, en particulier quant aux interactions sociales.

La médecine de réadaptation devrait se faire sous la direction d'un médecin spécialiste en médecine interne générale, travaillant en interdisciplinarité avec une équipe multidisciplinaire sur la base d'objectifs prédéfinis et négociés avec le patient. C'est le modèle théorique CIF, soit de la Classification internationale $\mathrm{du}$ fonctionnement, du handicap et de la santé [4] qui est utilisé comme moyen d'évaluer les déficits fonctionnels et leurs répercussions sur les activités quotidiennes.

La garantie et l'adéquation des prestations nécessitent un système de management de la qualité. Les critères de qualité doivent être simples, applicables, le moins contraignants possible tout en visant l'efficience. Nous sommes convaincus que les critères de qualité doivent non seulement représenter un standard minimal, mais aussi favoriser le développement de la spécialité médicale de la réadaptation en médecine interne.

\section{Définition de la réadaptation en médecine interne}

La réadaptation en médecine interne générale relève d'un processus orienté santé et autonomie, comprenant l'ensemble des mesures coordonnées sur le plan médical, éducatif et social. Elle doit permettre au patient de surmonter ses déficits ou handicaps, afin qu'il retrouve un fonctionnement physique, psychologique et social optimal. Alors il pourra redonner du sens à sa vie en retrouvant sa place dans la société.

Cette définition ne manque pas de provoquer des chevauchements avec les différents types de réadaptation qu'elle soit gériatrique, oncologique ou centrée sur un organe spécifique comme la réadaptation pulmonaire ou cardiaque par exemple. D'autre part, le risque de «morcellement» du patient entre différents spécialistes est élevé, même s'il est évident qu'une collaboration multidisciplinaire est nécessaire en fonction de la complexité de certaines pathologies. Le spécialiste en médecine interne générale a un rôle très important, non seulement comme ressource à part entière, mais aussi pour faire le lien entre les différents partenaires de soins.

\section{Indicateurs}

\section{Indicateurs d'admission}

L'indication à un programme de réadaptation dépend du degré de l'atteinte physique, du potentiel de récupération et de la capacité du patient à s'impliquer dans un tel projet de soins. Ces différents indicateurs modulent les objectifs qui doivent rester réalistes au risque de démotiver le patient comme son entourage.

Les indicateurs spécifiques peuvent inclure:

- Les maladies relevant de la médecine interne qui restreignent l'implication du patient et dont l'aptitude à la réadaptation reste présente.

- L'accumulation de plusieurs maladies et déficiences actives auxquels se surajoutent une intervention chirurgicale ou une atteinte organique aiguë qui décompensent l'état plus ou moins stable du patient.

- Toute limitation des activités de la vie quotidienne, des relations interpersonnelles, sociales, de l'instruction, de la formation, qui fragilisent le patient multimorbide.

Quant au choix d'une réadaptation stationnaire ou ambulatoire, la prise en compte des aspects tech- 
niques, de la capacité d'accueil en terme de personnel, d'infrastructures et de coûts globaux, doit être méticuleusement analysée.

Chaque institution de réadaptation doit définir ses propres critères en fonction de ses moyens, de ses compétences et de son expertise. L'indication et le choix entre institutions ambulatoires ou hospitalières peut s'inspirer du schéma [5] de la réhabilitation oncologique en Suisse (fig. 1).

\section{Indicateurs de processus}

- L'institution doit disposer de procédures cliniques adaptées à la clientèle.

- Le déroulement de l'admission, la définition des objectifs de soins et la planification de la sortie doit être documenté.

- Des instruments efficaces pour coordonner les processus sont nécessaires.

- Les objectifs de soins retenus sont documentés individuellement pour chaque patient.

- Les conseils médicaux donnés par des spécialistes (orthopédiste, cardiologue, pneumologue, etc. ) font l'objet d'un consilium.

- Une équipe apte à faire face aux urgences doit être disponible. Elle garantit les premiers secours, dont les mesures de réanimation (Basic Life Support) en l'espace d'une minute.

- Des systèmes de communication appropriés (mobile, dossier électronique consultable à distance, etc.) doivent faciliter le travail du médecin de garde ou de piquet, afin d'engager les mesures d'urgence.
- Le service médical doit être, à toute heure, auprès du patient dans les 15 minutes si nécessaire.

- L'institution procède régulièrement à l'évaluation des résultats, à la mise à jour des procédures de qualité, etc. ...

Spécialement pour les institutions stationnaires:

- Des adaptations journalières des thérapies sont possibles.

- L'accès à des examens complémentaires (radiographie, laboratoire, ECG standard, ECG d'effort, ECG longue durée, spirométrie, etc.) est garanti.

- Des examens spécialisés comme l'IRM et le PET scan sont accessibles raisonnablement en terme de proximité.

Ressources humaines (institutions stationnaires)

- La direction médicale occupe un poste d'au moins 80\%.

- La direction médicale dispose soit:

- d'un titre de spécialiste en médecine interne générale et 2 ans d'expérience en médecine de réadaptation dans une institution reconnue.

- d'un titre de spécialiste en médecine physique et réadaptation et 3 ans d'expérience en médecine interne générale dans une institution reconnue.

- La formation continue de la SSMI et de la SSMPR est accomplie.

- Alternative: Les qualifications ci-dessus sont disponibles parmi l'équipe des médecins cadres et ces médecins ont un poste fixe d'au moins $50 \%$.

\section{Figure 1}

Algorithme pour la prise de décision stationnaire ou ambulatoire.

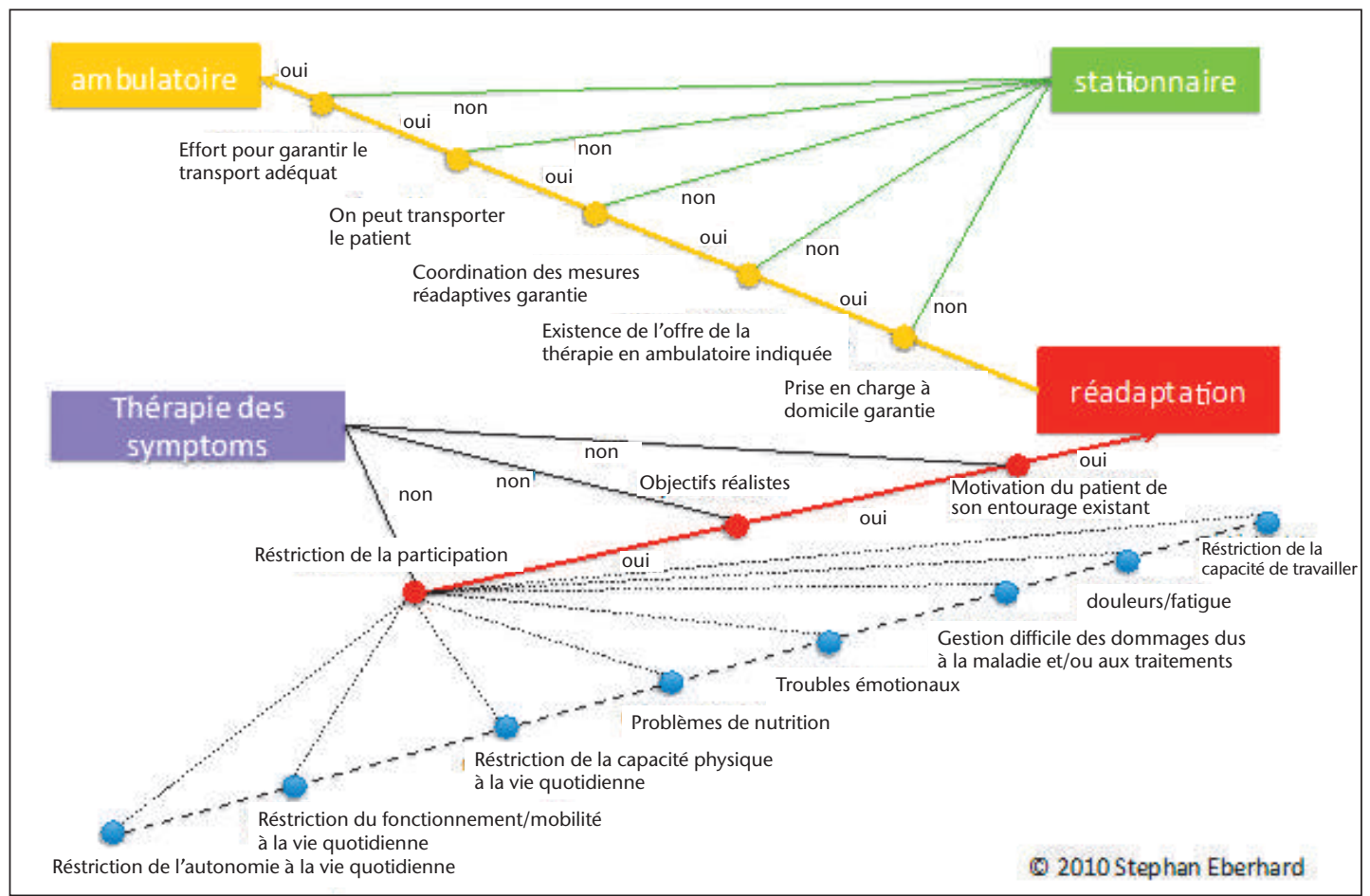


- La formation continue est accomplie.

- Les soins disposent d'un niveau Bachelor.

- Physiothérapie

- Ergothérapie

- Psychologie clinique

- Service social

- Diététique

- Cuisinier diététique

Selon le type de patient, les compétences suivantes doivent être disponibles dans la clinique de réadaptation ou en coopération avec d'autres institutions:

- Consultation de stomatologie

- Consultation de diabétologie

- Logopédie/réadaptation de la sphère oro-pharyngienne

- Chausseur orthopédiste/technicien orthopédiste

- Travailleur social

- Aumônerie

\section{Offres de traitements de base garantie (particulièrement en stationnaire)}

- Prise en charge continue des patient(e)s par le spécialiste en médecine interne générale

- Au moins un poste d'infirmier diplômé est occupé $24 \mathrm{~h}$ par jour

- Les soins invasifs sont possibles (perfusions, nutrition entérale, etc.)

- Physiothérapie individuelle et thérapies de groupes

- Ergothérapie individuelle et thérapies de groupes

- Thérapies manuelles

- Consultation nutritionnelle, thérapie individuelle et de groupes

- Service social

Selon le type d'institution d'autres prestations peuvent êtres mises à disposition

\section{Critères de résultats}

L'institution de réhabilitation définit les résultats à obtenir et les évalue pour chaque patient.

Exemple de critères:

- $\quad$ Test de marche des 6 minutes

- Timed-up-and-go-test

- Quotation de la capacité de participation (parex. WHODAS II) [6]

- Quotation des objectifs atteints en terme de participation (selon ANQ) [7]

Selon le type d'institution, d'autres grilles d'évaluation peuvent être utilisées.

\section{Conclusion}

En réadaptation en général et en médecine interne en particulier, il y a un gros potentiel d'amélioration du sentiment qu'a le patient de sa santé, en minimisant les restrictions quant à sa capacité de s'impli- quer dans un programme de soin. En revanche, le «morcellement» du patient va à l'opposé d'une prise en charge bio-psycho-sociale et spirituelle. La médecine interne générale de réadaptation considère toujours l'être humain comme une entité, ce qui s'oppose à une réadaptation axée sur un organe spécifique. Elle se distingue aussi par la capacité à intégrer à la fois les diagnostiques des différentes disciplines médicales somatiques et ceux psychiatriques dans un seul concept de traitement $[8,9]$.

Au cours des prochaines années, il serait souhaitable de cibler les efforts de recherche sur le pôle «médecine interne générale-réadaptation». Une des pistes de recherche clinique pourrait se faire sur l'évaluation des résultats. Jusqu'à présent, l'état de la recherche n'a que peu investigué ce pôle, c'est pourquoi les recommandations sont issues de consensus d'experts. Cependant, par analogie, des conclusions pourraient déjà être puisées dans des études existantes provenant de la réadaptation oncologique.

Ce document se veut une base de discussions, et aussi comme appel à toutes les sociétés de spécialistes (médicaux et paramédicaux) pour construire ensemble la réadaptation du futur.

Une nouvelle façon de voir ce domaine médical est nécessaire, particulièrement du fait de la pénurie des médecins, des exigences médico-légales, des pressions des politiciens et des assureurs.

L'objectif commun devrait être l'accessibilité à un réadaptation de qualité, ouverte à tous les patients de notre pays qui en ont besoin, que cela soit de manière ambulatoire ou stationnaire.

\section{Références}

1 Zürcher Spitalplanung 2012. Teil 2: Strukturbericht. www.gd.zh.ch/internet/gesundheitsdirektion/de/ themen/behoerden/spitalplanung_2012/strukturbericht.html\#subtitle-content-internet-gesundheitsdirektion-de-themen-behoerden-spitalplanung_2012 strukturbericht-jcr-content-contentPar-downloadfolder (23.9.2011)

2 Karrer W et al. Die Internistische Rehabilitation. Bull Méd Suisses. 2007;88(40):1672-4

3 Programme de formation postgraduée «médecine interne générale». $1^{\mathrm{er}}$ janvier 2011.

4 www.who.int/classifications/icf/en/

5 Eberhard S. Réadaptation: Programme national contre le cancer pour la Suisse 2011-2015. p. 148-61.

6 www.who.int/classifications/icf/whodasii/en/

7 www.anq.ch

8 Bauer W et al. Political issues in internal medicine in Europe: A posoition paper. European Journal of Internal Medicine. 2005;16:214-7.

9 Palsson R et al. Core competencies oft he European internist: A discussion paper. European Journal of Internal Medicine. 2007;18:104-8. 